\title{
Boundary segmentation for fluorescence microscopy using steerable filters
}

David Joon Ho, Paul Salama, Kenneth W. Dunn, Edward J. Delp

David Joon Ho, Paul Salama, Kenneth W. Dunn, Edward J. Delp, "Boundary segmentation for fluorescence microscopy using steerable filters," Proc. SPIE 10133, Medical Imaging 2017: Image Processing, 101330E (24 February 2017); doi: 10.1117/12.2254627

SPIE. Event: SPIE Medical Imaging, 2017, Orlando, Florida, United States 


\title{
Boundary Segmentation For Fluorescence Microscopy Using Steerable Filters
}

\author{
David Joon $\mathrm{Ho}^{\mathrm{a}}$, Paul Salama ${ }^{\mathrm{b}}$, Kenneth W. Dunn ${ }^{\mathrm{c}}$, and Edward J. Delp ${ }^{\mathrm{a}}$ \\ ${ }^{a}$ Video and Image Processing Laboratory, School of Electrical and Computer Engineering, \\ Purdue University, West Lafayette, Indiana 47907 U.S.A. \\ ${ }^{\mathrm{b}}$ Department of Electrical and Computer Engineering, Indiana University-Purdue University, \\ Indianapolis, Indiana 46202 U.S.A. \\ ${ }^{\mathrm{c}}$ Division of Nephrology, School of Medicine, Indiana University, Indianapolis, Indiana 46202 \\ U.S.A.
}

\begin{abstract}
Fluorescence microscopy is used to image multiple subcellular structures in living cells which are not readily observed using conventional optical microscopy. Moreover, two-photon microscopy is widely used to image structures deeper in tissue. Recent advancement in fluorescence microscopy has enabled the generation of large data sets of images at different depths, times, and spectral channels. Thus, automatic object segmentation is necessary since manual segmentation would be inefficient and biased. However, automatic segmentation is still a challenging problem as regions of interest may not have well defined boundaries as well as non-uniform pixel intensities. This paper describes a method for segmenting tubular structures in fluorescence microscopy images of rat kidney and liver samples using adaptive histogram equalization, foreground/background segmentation, steerable filters to capture directional tendencies, and connected-component analysis. The results from several data sets demonstrate that our method can segment tubular boundaries successfully. Moreover, our method has better performance when compared to other popular image segmentation methods when using ground truth data obtained via manual segmentation.
\end{abstract}

Keywords: fluorescence microscopy, image segmentation, steerable filters, tubule boundary

\section{INTRODUCTION}

In recent years, the advancement in fluorescence microscopy has enabled biologists to visualize subcellular structures in living cells or living animals [1], [2]. With the advancement in fluorescence microscopy, image segmentation methods have become important, since segmentation is prerequisite to any kind of quantitation. Due to the volume of data manual segmentation becomes prohibitive. Automatic segmentation methods are thus necessary in order to analyze fluorescence microscopy images in an unbiased and efficient manner [3], [4]. Automatic image segmentation of microscopy images can be challenging, since region boundaries are not well defined. In addition, voxels belonging to a common object/region may have disparate intensities.

Our data consist of image volumes collected from rat kidney and liver samples using two-photon microscopy. The images of rat kidney are labeled with fluorescently labeled phalloidin (which labels filamentous actin). The fluorescence of phalloidin labels two tissue structures, the basement membrane of the tubules and the brush border (present in the tubule lumen) of the proximal tubules. Our segmentation goal is to distinguish tubule borders from the brush border in order to identify the boundaries of renal tubules in three-dimensions, in order to characterize the tubular lumens of a single nephron in the kidney. The liver sample is labeled with a fluorescent tomato lectin, which labels cell boundaries and endothelia. Our segmentation goal for the liver data is to highlight blood vessels and cell-cell junctions, in order to quantitatively characterize the vascular space and hepatocytes.

Many image segmentation methods have been described for microscopy data. These include snakes or active contours [4], [5] that evolve curves by minimizing a cost function or snake energy. The performance of these

This work was supported by a George M. O'Brien Award from the National Institutes of Health NIH/NIDDK P30 DK079312.

Medical Imaging 2017: Image Processing, edited by Martin A. Styner, Elsa D. Angelini, Proc. of SPIE Vol. 10133, 101330E · C 2017 SPIE · CCC code: 1605-7422/17/\$18 · doi: 10.1117/12.2254627 
techniques is strongly dependent on the selection of an initial curve or curves. While manually choosing the initial curves or points may yield good results, it can be time consuming and laborious.

In [6], an automatic initialization method based on estimating an external energy field by solving Poisson's equation is described. Nonetheless, the performance of snake-like methods tends to deteriorate when the regions of interest do not have well defined or blurred boundaries. To circumvent this [7] describes the use of active contours combined with multiresolution methods, multiscale based smoothing, and region growing to segment fluorescence microscopy images which contain punctate patterns. Similarly, in [8], a fully automatic segmentation method using multiple active surfaces, a penalty function that penalizes overlaps, and a volume conservation constraint is described. Alternatively, [9] uses steerable filters to detect 2D features for better orientation selectivity, while [10] describes a discrete region competition method when the number of regions is unknown. More recently, a method known as Squassh [11], [12] that is based on object detection and segmentation using region competition and object-based colocalization, was developed for the segmentation and quantification of subcellular shapes. However, these methods tend to not properly segment regions of interest with inhomogeneous pixel/voxel intensities.

There are also methods that were developed specifically for segmenting tubular-like structures, namely blood vessels, in magnetic resonance angiography (MRA) or computed tomography (CT). [13] developed a method for tracing the centerline of tubular structures using a three-dimensional minimal path with minimum user interaction. Alternatively, [14] describes a 3D deformable model that represents a centerline coupled to a vessel surface, while [15] utilizes geodesic active contours of codimension two to automatically segment vessel surfaces. Li and Yezzi [16] combine minimal path [13] with active contours ([14], [15]) to simultaneously estimate centerlines and vessel surfaces of tubular structures by modeling a vessel as a $4 \mathrm{D}$ curve comprising the three-dimensional centerline of the vessel and its one-dimensional radius which corresponds to its surface. Several advanced methods have been developed using the 4D model [17], [18] but they still require manual initialization due to the complex structures. Additionally, these techniques may not successfully segment tubule boundaries, as exhibited in our data, since they do not distinguish between tubule boundaries and lumen.

A method that can automatically segment tubule boundaries in the presence of blur and the inhomogeneity exhibited by microscopy images is thus desired. In this paper we present a tubule segmentation method for fluorescence microscopy images that is able to segment tubular boundaries in the presence of intensity inhomogeneities and blur using adaptive histogram equalization, foreground/background segmentation, steerable filters [19], and connected-component analysis. Directional information obtained from steerable filters facilitates the segmentation of tubule and cell boundaries.

\section{PROPOSED METHOD}

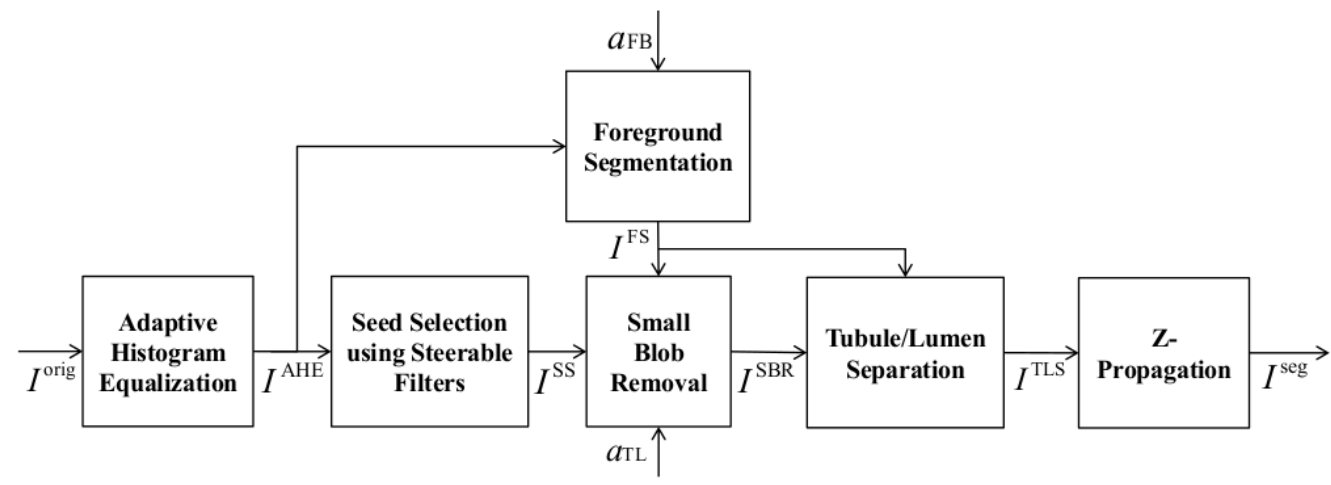

Figure 1. Block Diagram of the Proposed Method

Figure 1 is a block diagram of the proposed method. Adaptive histogram equalization is first applied to the original stack of images for enhancement purposes. The histogram equalized images are then segmented 
by a foreground/background technique described below. Three-dimensional steerable filters are also used to generate potential seeds that will be used to identify and grow tubule boundaries. This is followed by a small blob removal stage through which undesired seeds are removed and the remaining seeds are used to separate tubule boundaries from lumen in images using two-dimensional connected components. Finally, the results for each image are correlated along the z-direction to remove any mis-labeled voxels.

We shall denote a 3D image volume of focal planes of size of $X \times Y \times Z$ by $I$, and the $p^{\text {th }} 2 \mathrm{D}$ focal plane image of size of $X \times Y$ pixels in $I$ where $p \in\{1, \ldots, Z\}$ by $I_{z_{p}}$. For example, $I^{\text {orig }}$ is a 3D original image volume and $I_{z_{95}}^{\text {orig }}$ is a $2 \mathrm{D}$ image of the 95 th focal plane.

\subsection{Histogram Equalization and Foreground Segmentation}

Since fluorescence microscopy images have non-uniform intensities, with low intensities being visible in the vicinity of the image boundaries, a pre-processing step is used to enhance regions close to the boundaries. This is achieved via $3 \mathrm{D}$ adaptive histogram equalization that employs a $17 \times 17 \times 9$ rectangular window, where voxels near the stack boundaries are mirrored past the boundaries. Since the resolution along the z-direction is smaller than along the $\mathrm{x}$ and $\mathrm{y}$-directions, the window dimensions were chosen accordingly. This step is used since it brightens only "foreground pixels" locally.

Foreground segmentation, in which the images are separated into "foreground" (F) and "background" (B) regions, is then done next. The foreground/background regions are determined by:

$$
I^{\mathrm{FS}}(\mathbf{v})= \begin{cases}255, & \text { if } I^{\mathrm{AHE}}(\mathbf{v})>255 \cdot\left(1-a_{\mathrm{FB}}\right) \\ 0, & \text { otherwise }\end{cases}
$$

where $I^{\mathrm{AHE}}$ is the histogram equalized image volume, $\mathbf{v}=(x, y, z)$ is a voxel location, and $a_{\mathrm{FB}}$ denotes the ratio of the number of voxels in the foreground to the total number of voxels. The value of $a_{\mathrm{FB}}$ is changed according to the desired size of foreground $(\mathbf{F})$ and background $(\mathbf{B})$ regions. For example, if the value of $a_{\mathrm{FB}}$ is increased, then the number of voxels belonging to foreground region may also increase.

\subsection{Seed Selection Using Steerable Filters}

In order to distinguish tubule boundaries within the foreground, "seeds" for constructing/growing each potential tubule boundary are needed. Since tubule boundaries tend to be thinner than lumen, seed selection is accomplished through the use of steerable filters [19] having various orientations that are capable of capturing the directional tendencies of thin regions. A property of steerable filters is that they can be synthesized as a linear combination of basis filters [19]. The advantage of steerable filters is that they can be used to detect local orientation of edges [20].

A 3D steerable filter with orientation angles $\theta$ and $\phi$ (see Figure 2), $h^{\theta, \phi}$, can be obtained as:

$$
\begin{aligned}
h^{\theta, \phi}(\mathbf{v})= & \sin ^{2}(\phi) \cos ^{2}(\theta) g_{x x}(\mathbf{v})+\sin ^{2}(\phi) \sin ^{2}(\theta) g_{y y}(\mathbf{v})+\cos ^{2}(\phi) g_{z z}(\mathbf{v})-\sin ^{2}(\phi) \sin (2 \theta) g_{x y}(\mathbf{v}) \\
& -\sin (2 \phi) \cos (\theta) g_{x z}(\mathbf{v})-\sin (2 \phi) \sin (\theta) g_{y z}(\mathbf{v})
\end{aligned}
$$

where $\mathbf{v}=(x, y, z)$ denotes voxel location, $\theta$ an angle in the xy-plane relative to the $\mathrm{x}$-axis, $\phi$ an angle relative to the z-axis, and $g(\mathbf{v})$ a Gaussian function given by $g(x, y, z)=e^{-\left(x^{2}+y^{2}+z^{2}\right)}$ with rectangular support that is $24 \times 24 \times 24$ in size. Allowing the values of $\theta$ and $\phi$ to vary between $0^{\circ}$ to $180^{\circ}$ in intervals of $22.5^{\circ}$ results in 64 steerable filters.

Generally, tubules have thin boundaries and voxel intensities on tubule boundaries are higher than voxel intensities in the background. Therefore, a voxel intensity function will be locally concave along the normal to a tubule boundary. Consequently, the second derivative of the intensity function will typically be small near tubule boundaries. We utilize this fact and initially generate 64 steerable filter responses, $R^{\theta, \phi}$, which are responses with orientation $\theta$ and $\phi$, respectively, of the 64 different steerable filters to the output of the adaptive histogram equalization step, $I^{\mathrm{AHE}}$, that is:

$$
R^{\theta, \phi}=I^{\mathrm{AHE}} * * h^{\theta, \phi}
$$




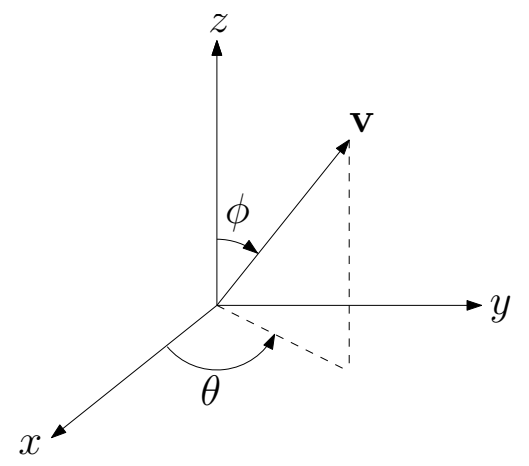

Figure 2. Spherical Coordinates

where ${ }^{* *}$ denotes $3 \mathrm{D}$ convolution.

Next we obtain the minimum responses, $I^{\mathrm{MR}}(\mathbf{v})=\min _{\theta, \phi} R^{\theta, \phi}(\mathbf{v}), I^{\theta}(\mathbf{v})=\underset{\theta}{\arg \min } R^{\theta, \phi}(\mathbf{v})$, and $I^{\phi}(\mathbf{v})=$ $\arg \min R^{\theta, \phi}(\mathbf{v})$. The smaller $I^{\mathrm{MR}}(\mathbf{v})$ is at voxel $\mathbf{v}$ the stronger directional tendency that voxel will have. Similarly, $\phi$ $I^{\theta}(\mathbf{v})$ and $I^{\phi}(\mathbf{v})$ provide the strong directional tendencies at voxel $\mathbf{v}$ along the $\theta$ and $\phi$ directions, respectively.

In order to decide how many voxels will be selected as seed candidates, we define the seed ratio, $r_{s}$, as the ratio of the number of seed candidates to the total number of voxels:

$$
r_{s}=\frac{\sum_{x=1}^{X} \sum_{y=1}^{Y} \sum_{z=1}^{Z} I^{\text {orig }}(x, y, z)}{255 X Y Z}
$$

where the size of the stack is $X \times Y \times Z$. The brighter the voxel intensities in $I^{\text {orig }}$ are the more voxels are selected as seed candidates. For each focal plane image, we obtain the $N_{s}=\left\lfloor r_{s} X Y\right\rfloor$ smallest values of $I_{z_{p}}^{\mathrm{MR}}$ and choose these voxels to be seed candidates. Therefore, we have $N_{s}$ seed candidates for each 2D image. The resulting image wherein seeds have been selected is denoted by $I_{z_{p}}^{\mathrm{SS}}$.

\subsection{Small Blob Removal and Tubule/Lumen Separation}

In the prior seed selection stage, seed candidates were selected, but not all candidates lie on tubule boundaries. Thus, it is necessary to remove seed candidates not belonging to tubule boundaries. Initially, all seeds not belonging to foreground sections in $I^{\mathrm{FS}}$ are removed. In the previous step, we created 3D stacks, $I^{\theta}(\mathbf{v})$ and $I^{\phi}(\mathbf{v})$, which contain the orientation of strong directional tendencies at $\mathbf{v}$. For each orientation pair $(\theta, \phi)$ on every $2 \mathrm{D}$ image, $I_{z_{p}}^{\mathrm{SS}}$, seed candidates having the orientation $(\theta, \phi)$ and that reside in a connected region of size (size here denotes the number of connected components) less than a blobsize threshold, $t_{b}$, given by $t_{b}=a_{\mathrm{TL}} r_{s} X Y Z$, are removed using 2D connected component analysis. Here $a_{\mathrm{TL}}$ is the ratio of the number of seed candidates in the largest blob in a lumen to the total number of seed candidates in the image. After removing small blobs in each orientation, the remaining seeds are selected to be final candidates denoted as $I^{\mathrm{SBR}}$.

Subsequent to small blob removal, tubule/lumen separation is performed based on the location of the final seeds in $I^{\mathrm{SBR}}$. Using 2D connected component analysis all foreground $(\mathbf{F})$ blobs on every focal plane image, $I_{z_{p}}^{\mathrm{FS}}$, that contain seeds are labeled as tubule boundaries $(\mathbf{T})$, whereas the remaining voxels in the foreground are labeled as lumen $(\mathbf{L})$. Here, the value of $a_{\mathrm{TL}}$ can be tuned according to the desired size of tubule boundaries $(\mathbf{T})$ and lumen $(\mathbf{L})$ regions. For example, if $a_{\mathrm{TL}}$ is increased, then $t_{b}$ will be increased and the number of final seed candidates will be reduced, which may result in smaller tubule regions in the foreground region.

\subsection{Z-Propagation Refinement}

We assume that sudden changes in the biological structure are unlikely to occur between adjacent slices along the z-direction and hence adjacent voxels along the z-direction will typically have the same label or belong to the same tubule boundary. Since some adjacent voxels along the z-direction may not have the same label, an 
extra post-processing step is necessary. We choose a set of voxels along the z-direction that have the same $(x, y)$ position. Recall that each voxel is labeled as $\mathbf{T}, \mathbf{L}$, or $\mathbf{B}$, representing tubule boundary, lumen, or background respectively. Next we divide this set into disjoint subsets such that each subset contains only voxels labeled as $\mathbf{T}$ or $\mathbf{L}$ (see Figure 3). For each such subset, a majority vote decides whether $\mathbf{T}$ or $\mathbf{L}$ is assigned to all pixels of that subset.

Figure 3 depicts a set of labeled voxels consisting of voxels belonging to a specific $(x, y)$ position but differing z-planes. This set is divided into multiple subsets separated by the voxels labeled as $\mathbf{B}$. In subset-1, most of the voxels are labeled as $\mathbf{L}$, therefore all the voxels from subset-1 will be labeled as $\mathbf{L}$, based on majority voting. In contrast, in subset-2 and subset-3, all voxels are labeled as $\mathbf{T}$, based on respective majority votes.

\section{Before z-propagation}

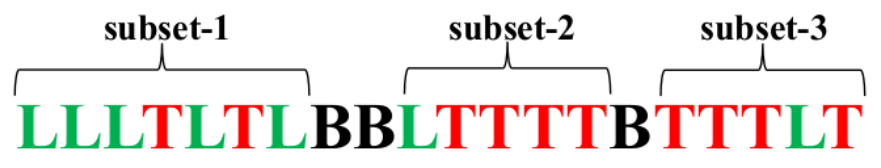

After z-propagation

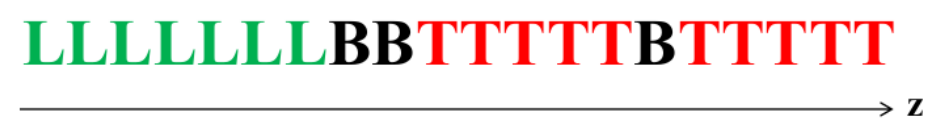

Figure 3. Example of Z-Propagation Refinement

\section{EXPERIMENTAL RESULTS}

We tested our method on microscopy data, denoted as Data-I*, consisting of $Z=512$ images of rat kidney where each image was $X=512 \times Y=512$ pixels in size. For this particular data $a_{\mathrm{FB}}$ was empirically found to be 0.2138 and $a_{\text {TL }}$ empirically determined to be $5.86 \times 10^{-7}$. Figure 4 shows the images obtained at each step for $I_{z_{95}}$ in Data-I. In addition, Figure 5 shows the results produced by the proposed method for several images located at various depths.

We compare the performance of the proposed method to several other segmentation methods used in microscopy images including active contours [5], Jacob and Unser's ImageJ plugin known as steerableJ [9], region competition [10], and Squassh [11], [12], as shown in Figure 6.

It was observed that in the case of active contours, which required the manual selection of initial curves for each tubule boundary, most of the initial curves evolved poorly although they were initialized very closely to the actual boundaries. This was observed to be the case since the tubule boundaries in the Data-I were not well defined. As can be observed SteerableJ detects not only tubule boundaries but also lumens although it failed to detect tubule boundaries located at image boundaries where the pixel intensities were low. As far as region competition was concerned it had no ability to distinguish between tubule boundary and lumen. Similarly Squassh also failed to distinguish between tubule boundary and lumen and it only segmented foreground regions located in the central area of the image.

All methods were also evaluated using reference data that was generated by manually segmenting the images. The metrics used in the evaluation were segmentation accuracy and the Type-I and Type-II error ratios. Here accuracy, Type-I error, and Type-II error ratios were defined as accuracy $=\frac{n_{\mathrm{TP}}+n_{\mathrm{TN}}}{n_{\text {total }}}$, Type-I error $=\frac{n_{\mathrm{FP}}}{n_{\text {total }}}$, Type-II error $=\frac{n_{\mathrm{FN}}}{n_{\text {total }}}$, where $n_{\mathrm{TP}}, n_{\mathrm{TN}}, n_{\mathrm{FP}}, n_{\mathrm{FN}}, n_{\text {total }}$ denote the number of true-positives (pixels on tubule boundary that are correctly labeled as belonging to a tubule boundary), true-negatives (lumen/background pixels that are correctly labeled as either lumen/background), false-positives (number of pixels belonging to lumen/background that are wrongly labeled as belonging to tubule boundary), false-negatives (number of pixels

${ }^{*}$ Data-I was provided by Malgorzata Kamocka of Indiana University and was collected at the Indiana Center for Biological Microscopy. 


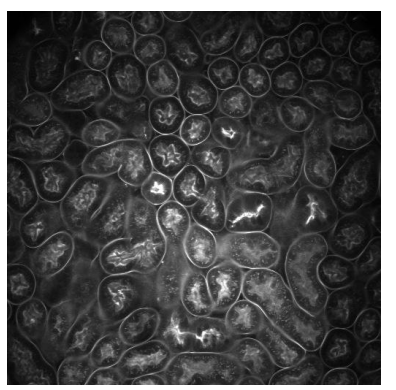

(a)

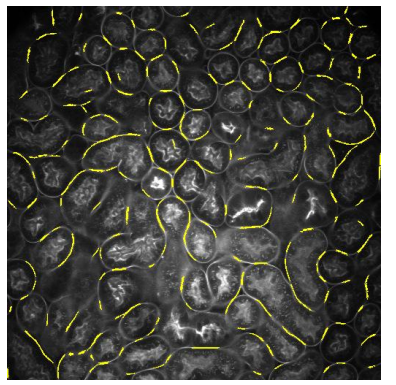

(e)

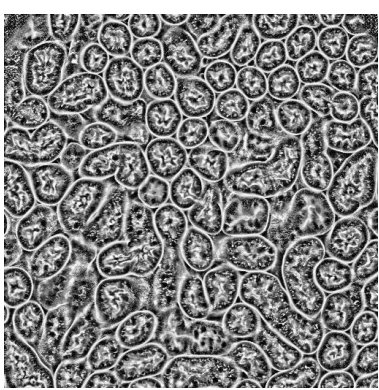

(b)

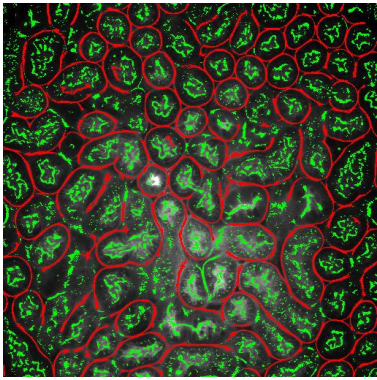

(f)

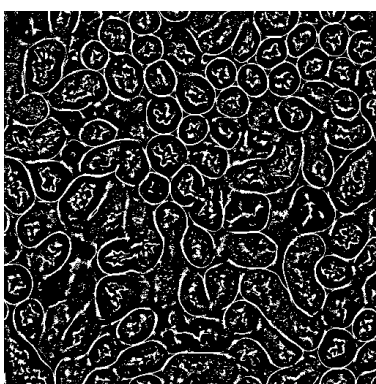

(c)

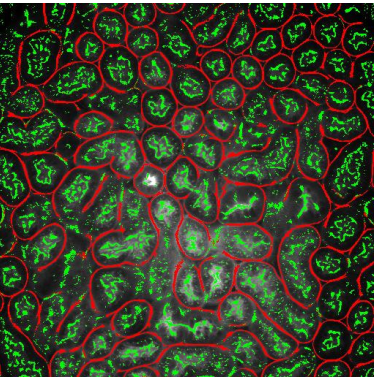

(g)

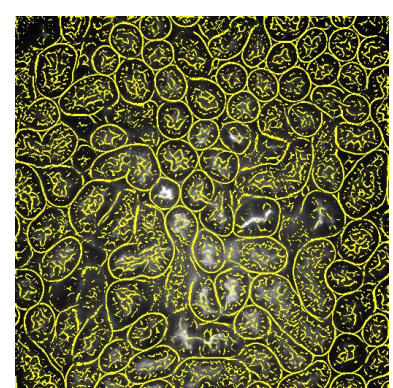

(d)

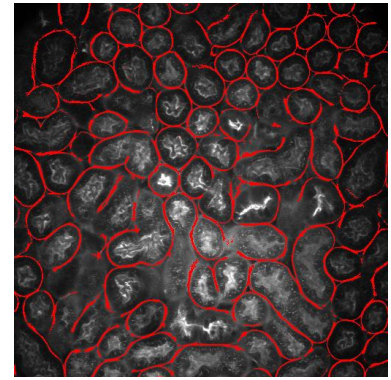

(h)

Figure 4. Original and output images for $I_{z_{95}}$ in Data-I: (a) original image, $I_{z_{95}}^{\text {orig }}$, (b) adaptive histogram equalized image, $I_{z_{95}}^{\mathrm{AHE}},(\mathrm{c})$ foreground segmented image, $I_{z_{95}}^{\mathrm{FS}},(\mathrm{d})$ seeds before small blob removal, $I_{z_{95}}^{\mathrm{SS}}$, (e) seeds after small blob removal, $I_{z_{95}}^{\mathrm{SBR}}$, (f) tubule/lumen separation before z-propagation, $I_{z_{95}}^{\mathrm{TLS}}$, (g) tubule/lumen separation after z-propagation, and (h) tubule boundaries only after z-propagation, $I_{z_{95}}^{\text {seg }}$

on tubule boundaries that are wrongly labeled as either lumen/background), and the total number of pixels, respectively. As can be observed from Table 1 our method outperformed the other techniques. It is to be noted that the parameters of the aforementioned techniques were tuned to produce the best results.

Table 1. Accuracy, Type-I and Type-II errors for known methods and our method on $I_{z_{95}}$ of the Data-I

\begin{tabular}{|c|c|c|c|}
\hline & Accuracy & Type I error & Type II error \\
\hline Active Contours & $85.56 \%$ & $1.83 \%$ & $12.61 \%$ \\
\hline steerableJ & $78.40 \%$ & $12.16 \%$ & $9.44 \%$ \\
\hline Region Competition & $76.58 \%$ & $12.99 \%$ & $10.43 \%$ \\
\hline Squassh & $68.45 \%$ & $22.33 \%$ & $9.22 \%$ \\
\hline Proposed Method & $90.86 \%$ & $1.63 \%$ & $7.51 \%$ \\
\hline
\end{tabular}

Table 2. Accuracy, Type-I and Type-II errors for known methods and our method on $I_{z_{17}}$ of the Data-II

\begin{tabular}{|c|c|c|c|}
\hline & Accuracy & Type-I error & Type-II error \\
\hline Active Contours & $78.76 \%$ & $0.56 \%$ & $20.68 \%$ \\
\hline steerableJ & $82.44 \%$ & $4.18 \%$ & $13.38 \%$ \\
\hline Region Competition & $69.50 \%$ & $11.64 \%$ & $18.86 \%$ \\
\hline Squassh & $85.61 \%$ & $10.09 \%$ & $4.30 \%$ \\
\hline Proposed Method & $86.35 \%$ & $6.62 \%$ & $7.03 \%$ \\
\hline
\end{tabular}

Our method was also tested on three liver datasets: Data-II, Data-III, and Data-IV ${ }^{\dagger}$, where $X=512$, $Y=512$, and $Z=36$ in the case of Data-II, $X=512, Y=512$, and $Z=1$ for Data-III, and $X=512, Y=512$, and $Z=156$ in the case of Data-IV. As shown in Figure 7, Figure 8, Figure 9 and observed from Table 2, Table

${ }^{\dagger}$ Data-II, Data-III, and Data-IV were provided by Sherry Clendenon and James Sluka of the Biocomplexity Institute, Indiana University at Bloomington. 


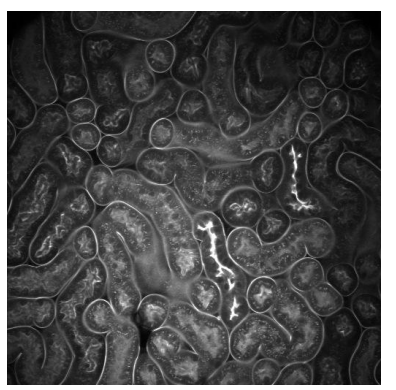

(a)

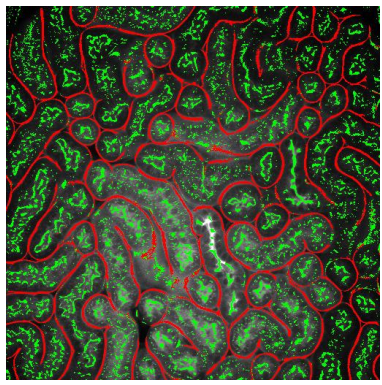

(e)

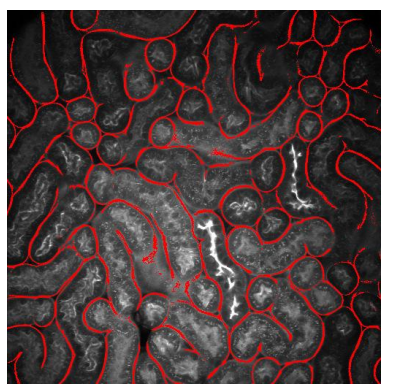

(i)

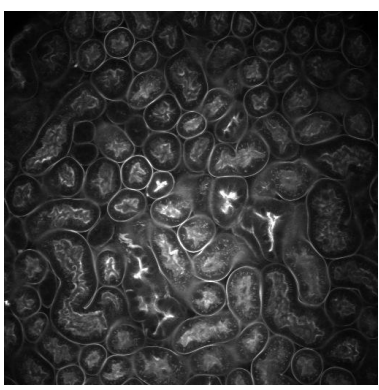

(b)

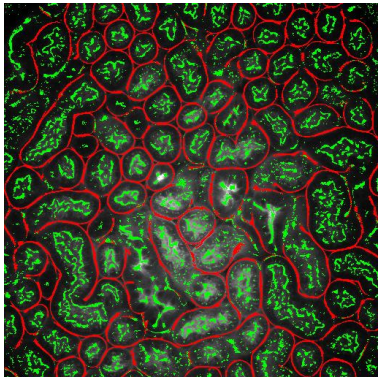

(f)

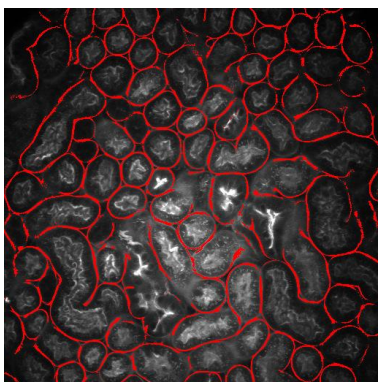

(j)

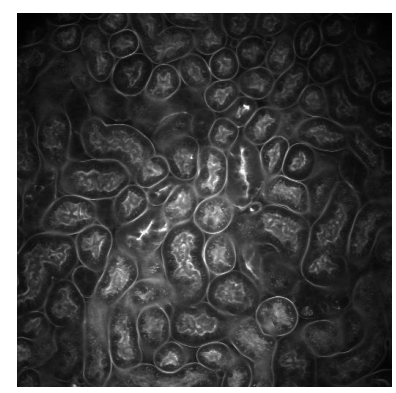

(c)

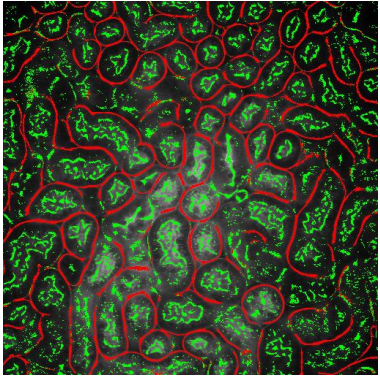

(g)

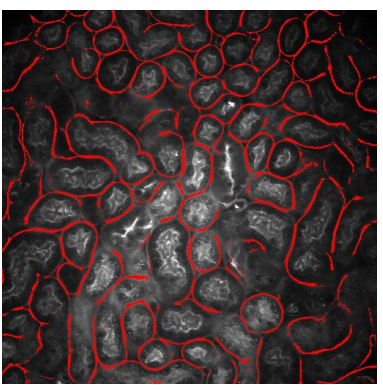

(k)

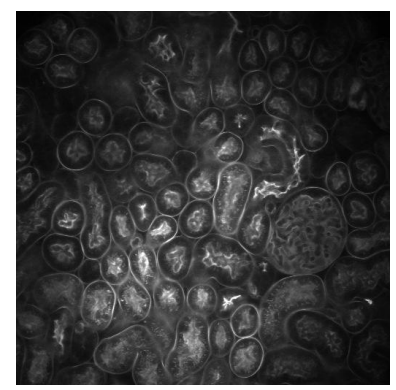

(d)

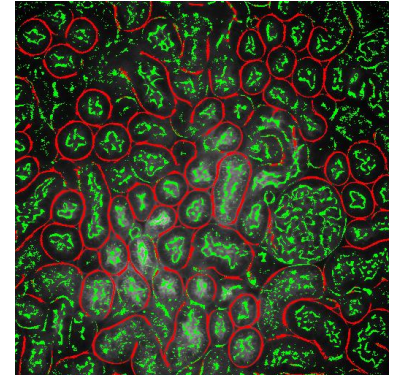

(h)

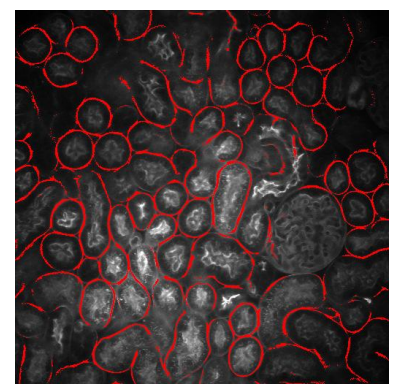

(l)

Figure 5. Described method on other focal planes in Data-I (a) $I_{z_{32}}^{\text {orig }}$ (b) $I_{z_{127}}^{\text {orig }}$ (c) $I_{z_{179}}^{\text {orig }}$ (d) $I_{z_{248}}^{\text {orig }}$ (e) $I_{z_{32}}^{\text {seg }}$ with tubule and lumen (f) $I_{z_{127}}^{\text {seg }}$ with tubule and lumen (g) $I_{z_{179}}^{\text {seg }}$ with tubule and lumen (h) $I_{z_{248}}^{\text {seg }}$ with tubule and lumen (i) $I_{z_{32}}^{\text {seg }}$ with tubule boundaries only (j) $I_{z_{127}}^{\text {seg }}$ with tubule boundaries only (k) $I_{z_{179}}^{\text {seg }}$ with tubule boundaries only (l) $I_{z_{248}}^{\text {seg }}$ with tubule boundaries only

Table 3. Accuracy, Type-I and Type-II errors for known methods and our method on $I_{z_{1}}$ of the Data-III

\begin{tabular}{|c|c|c|c|}
\hline & Accuracy & Type-I error & Type-II error \\
\hline Active Contours & $82.04 \%$ & $0.66 \%$ & $17.30 \%$ \\
\hline steerableJ & $84.68 \%$ & $3.52 \%$ & $11.80 \%$ \\
\hline Region Competition & $72.92 \%$ & $11.08 \%$ & $16.00 \%$ \\
\hline Squassh & $85.83 \%$ & $11.41 \%$ & $2.76 \%$ \\
\hline Proposed Method & $88.05 \%$ & $5.84 \%$ & $6.11 \%$ \\
\hline
\end{tabular}

3, and Table 4, our method successfully identified tubule and cell boundaries in various focal planes. In this case the values utilized for the parameters $a_{\mathrm{FB}}$ and $a_{\mathrm{TL}}$ were $a_{\mathrm{FB}}=0.27$ and $a_{\mathrm{TL}}=1.00 \times 10^{-5}$ for Data-II, $a_{\mathrm{FB}}=0.27$ and $a_{\mathrm{TL}}=1.50 \times 10^{-3}$ for Data-III, and $a_{\mathrm{FB}}=0.20$ and $a_{\mathrm{TL}}=6.41 \times 10^{-7}$ for Data-IV, respectively.

\section{CONCLUSIONS}

This paper described a method for segmenting tubule boundaries in a stack of microscopy images based on adaptive histogram equalization, foreground/background segmentation, steerable filters that generate directional 


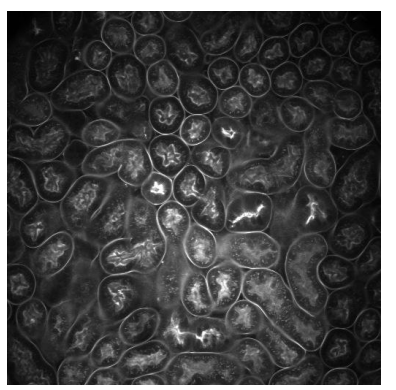

(a)

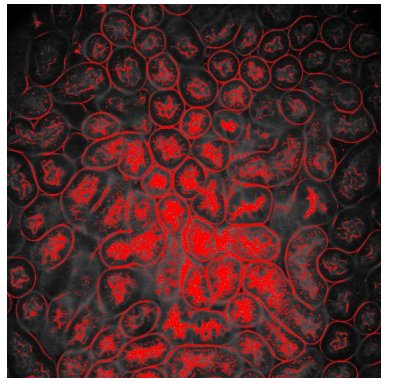

(e)

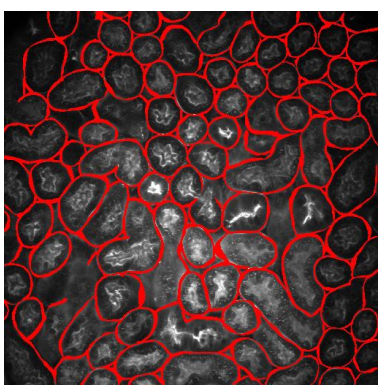

(b)

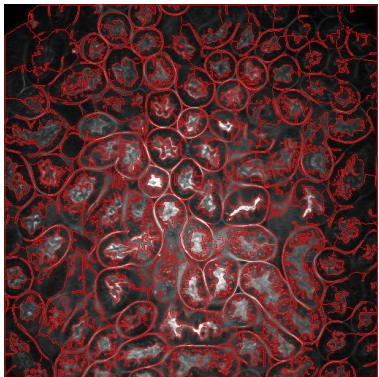

(f)

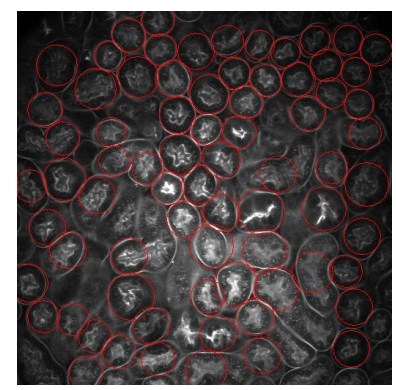

(c)

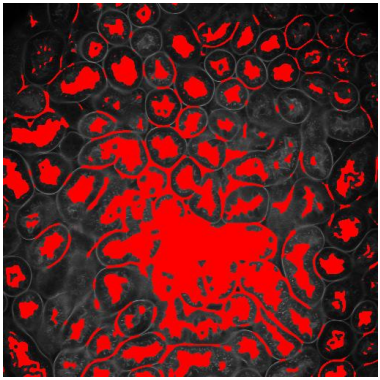

(g)

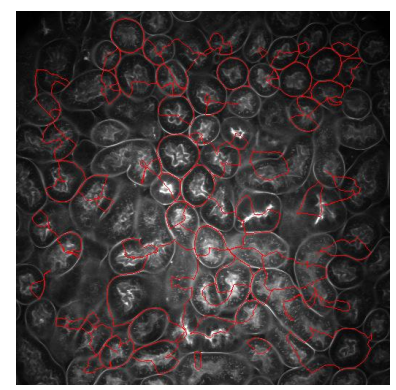

(d)

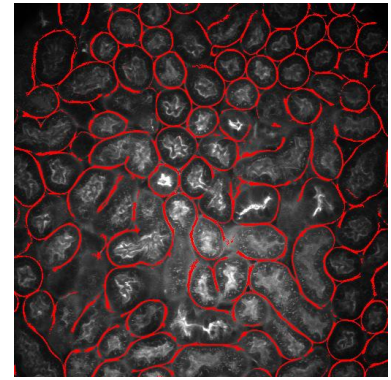

(h)

Figure 6. Comparison of the segmentation of $I_{z_{95}}$ in Data-I by the proposed method and other methods. (a) the original image, (b) reference segmentation, (c) active contours initial curves, (d) active contours final curves, (e) steerableJ segmentation outcome, (f) output image from region competition plugin, (g) Squassh segmented image, (h) outcome of the proposed method with tubule boundary only

Table 4. Accuracy, Type-I and Type-II errors for known methods and our method on $I_{z_{81}}$ of the Data-IV

\begin{tabular}{|c|c|c|c|}
\hline & Accuracy & Type-I error & Type-II error \\
\hline steerableJ & $87.38 \%$ & $4.18 \%$ & $8.44 \%$ \\
\hline Region Competition & $85.42 \%$ & $4.16 \%$ & $10.41 \%$ \\
\hline Squassh & $90.07 \%$ & $1.33 \%$ & $8.60 \%$ \\
\hline Proposed Method & $88.27 \%$ & $3.18 \%$ & $8.55 \%$ \\
\hline
\end{tabular}

information, and connected-components analysis. The results demonstrate that the described method outperformed other segmentation methods when used on a rat kidney data, and also had very good performance when used on several liver data. Future work will involve quantifying the characteristics of biological structures.

\section{REFERENCES}

[1] K. Dunn, R. Sandoval, K. Kelly, P. Dagher, G. Tanner, S. Atkinson, R. Bacallao, and B. Molitoris, "Functional studies of the kidney of living animals using multicolor two-photon microscopy," American Journal of Physiology-Cell Physiology, vol. 283, no. 3, pp. C905-C916, September 2002.

[2] C. Vonesch, F. Aguet, J. Vonesch, and M. Unser, "The colored revolution of bioimaging," IEEE Signal Processing Magazine, vol. 23, no. 3, pp. 20-31, May 2006.

[3] K. Lorenz, P. Salama, K. Dunn, and E. Delp, "Three dimensional segmentation of fluorescence microscopy images using active surfaces," Proceedings of the IEEE International Conference on Image Processing, pp. 1153-1157, September 2013, Melbourne, Australia.

[4] R. Delgado-Gonzalo, V. Uhlmann, D. Schmitter, and M. Unser, "Snakes on a plane: A perfect snap for bioimage analysis," IEEE Signal Processing Magazine, vol. 32, no. 1, pp. 41-48, January 2015.

[5] B. Li and S. T. Acton, "Active contour external force using vector field convolution for image segmentation," IEEE Transactions on Image Processing, vol. 16, no. 8, pp. 2096-2106, August 2007.

[6] B. Li and S. T. Acton, "Automatic active model initialization via poisson inverse gradient," IEEE Transactions on Image Processing, vol. 17, no. 8, pp. 1406-1420, August 2008. 


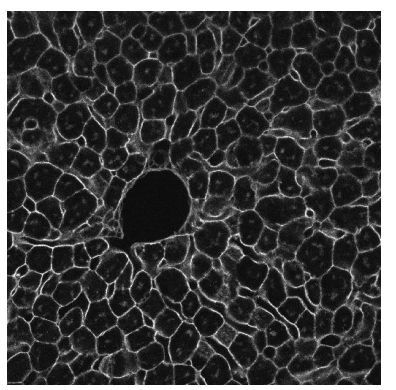

(a)

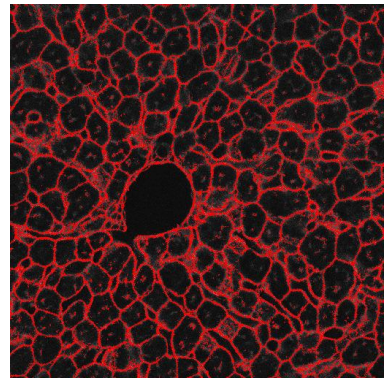

(e)

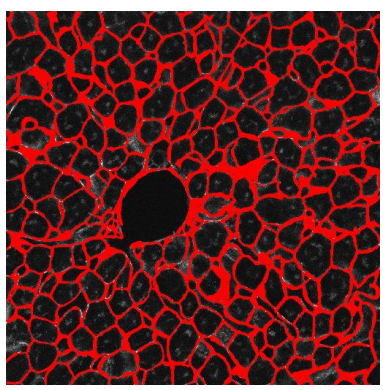

(b)

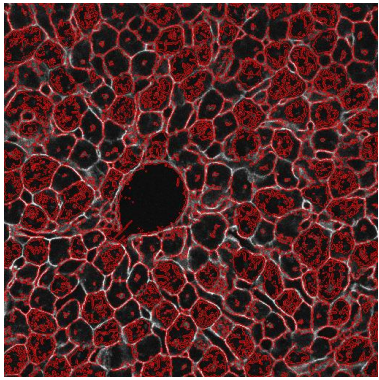

(f)

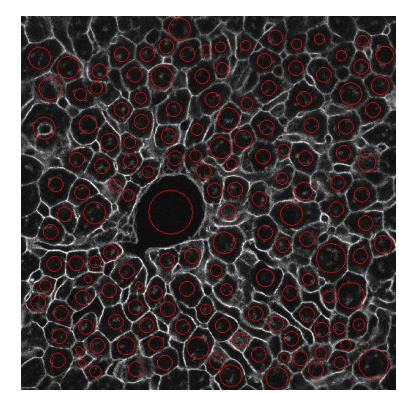

(c)

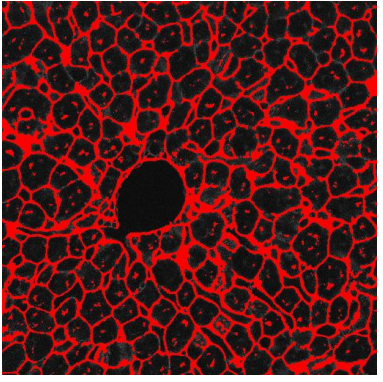

(g)

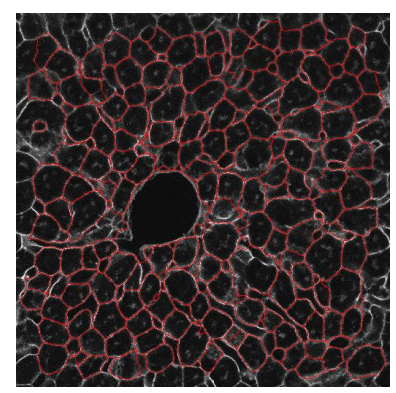

(d)

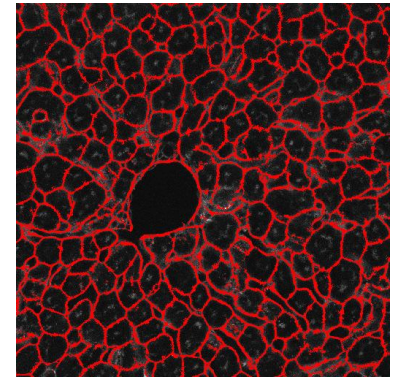

(h)

Figure 7. Comparison of the segmentation of $I_{z_{17}}$ in Data-II by the proposed method and other methods. (a) the original image, (b) reference segmentation, (c) active contours initial curves, (d) active contours final curves, (e) steerableJ segmentation outcome, (f) output image from region competition plugin, (g) Squassh segmented image, (h) outcome of the proposed method with tubule boundary only

[7] G. Srinivasa, M. C. Fickus, Y. Guo, A. D. Linstedt, and J. Kovacevic, "Active mask segmentation of fluorescence microscope images," IEEE Transactions on Image Processing, vol. 18, no. 8, pp. 1817-1829, August 2009.

[8] A. Dufour, V. Shinin, S. Tajbakhsh, N. Guillen-Aghion, J. C. Olivo-Marin, and C. Zimmer, "Segmenting and tracking fluorescent cells in dynamic 3-D microscopy with coupled active surfaces," IEEE Transactions on Image Processing, vol. 14, no. 9, pp. 1396-1410, September 2005.

[9] M. Jacob and M. Unser, "Design of steerable filters for feature detection using canny-like criteria," IEEE Transactions on Pattern Analysis and Machine Intelligence, vol. 26, no. 8, pp. 1007-1019, August 2004.

[10] J. Cardinale, G. Paul, and I. Sbalzarini, "Discrete region competition for unknown numbers of connected regions," IEEE Transactions on Image Processing, vol. 21, no. 8, pp. 3531-3545, August 2012.

[11] G. Paul, J. Cardinale, and I. Sbalzarini, "Coupling image restoration and segmentation: A generalized linear model/bregman perspective," International Journal of Computer Vision, vol. 104, no. 1, p. 6993, March 2013.

[12] A. Rizk, G. Paul, P. Incardona, M. Bugarski, M. Mansouri, A. Niemann, U. Ziegler, P. Berger, and I. Sbalzarini, "Segmentation and quantification of subcellular structures in fluorescence microscopy images using Squassh," Nature Protocols, vol. 9, no. 3, pp. 586-596, February 2014.

[13] T. Deschamps and L. D. Cohen, "Fast extraction of minimal paths in 3D images and applications to virtual endoscopy," Medical Image Analysis, vol. 5, no. 4, pp. 281-299, December 2001.

[14] A. F. Frangi, W. J. Niessen, R. M. Hoogeveen, T. van Walsum, and M. A. Viergever, "Model-based quantitation of 3-D magnetic resonance angiographic images," IEEE Transactions on Medical Imaging, vol. 18, no. 10, pp. 946-956, October 1999.

[15] L. M. Lorigo, O. D. Faugeras, W. E. L. Grimson, R. Keriven, R. Kikinis, A. Nabavi, and C.-F. Westin, "CURVES: Curve evolution for vessel segmentation," Medical Image Analysis, vol. 5, no. 3, pp. 195-206, September 2001.

[16] H. Li and A. Yezzi, "Vessels as 4-D curves: Global minimal 4-D paths to extract 3-D tubular surfaces and centerlines," IEEE Transactions on Medical Imaging, vol. 26, no. 9, pp. 1213-1223, September 2007. 


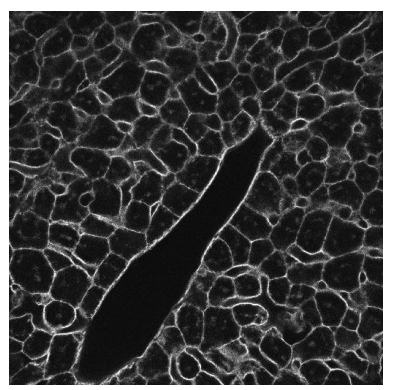

(a)

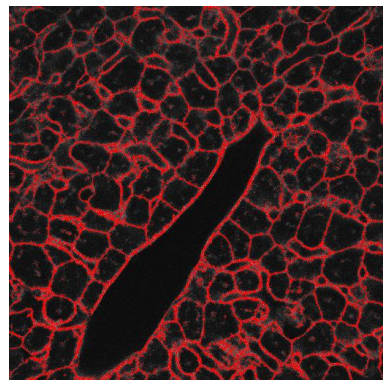

(e)

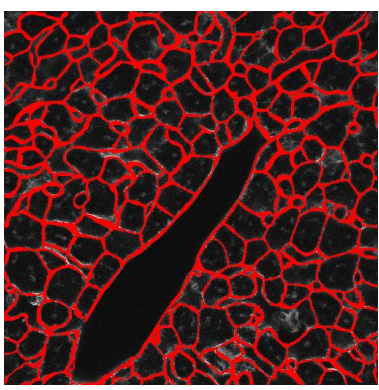

(b)

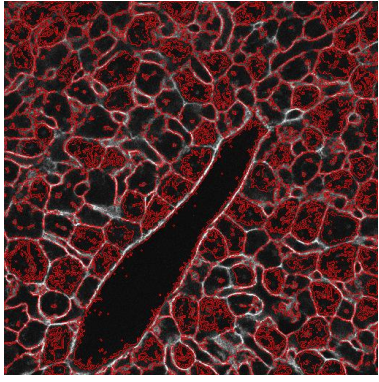

(f)

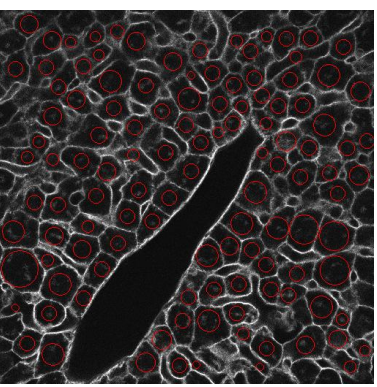

(c)

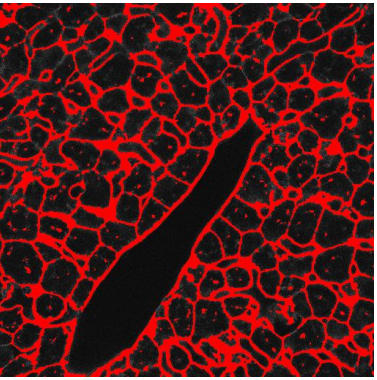

(g)

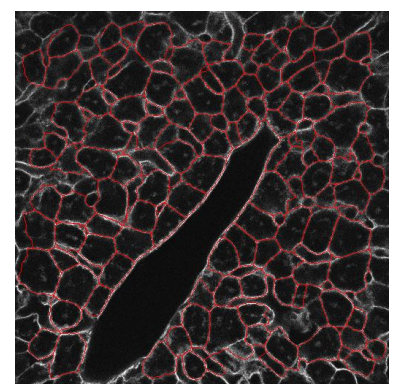

(d)

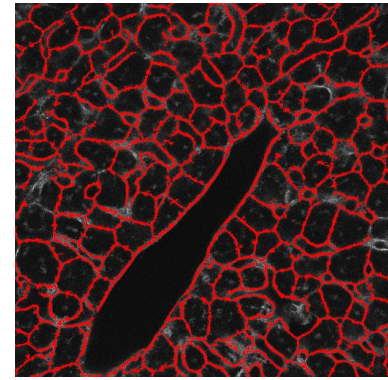

(h)

Figure 8. Comparison of the segmentation of $I_{z_{1}}$ in Data-III by the proposed method and other methods. (a) the original image, (b) reference segmentation, (c) active contours initial curves, (d) active contours final curves, (e) steerableJ segmentation outcome, (f) output image from region competition plugin, (g) Squassh segmented image, (h) outcome of the proposed method with tubule boundary only

[17] F. Benmansour and L. D. Cohen, "Tubular structure segmentation based on minimal path method and anisotropic enhancement," International Journal of Computer Vision, vol. 92, no. 2, pp. 192-210, March 2010.

[18] V. Mohan, G. Sundaramoorthi, and A. Tannenbaum, "Tubular surface segmentation for extracting anatomical structures from medical imagery," IEEE Transactions on Medical Imaging, vol. 29, no. 12, pp. 1945-1958, December 2010.

[19] W. Freeman and E. Adelson, "The design and use of steerable filters," IEEE Transactions on Pattern Analysis and Machine Intelligence, vol. 13, no. 9, pp. 891-906, September 1991.

[20] S. Jeong, Y. Tarabalka, and J. Zerubia, "Marked point process model for facial wrinkle detection," Proceedings of the IEEE International Conference on Image Processing, pp. 1391-1394, October 2014, Paris, France. 


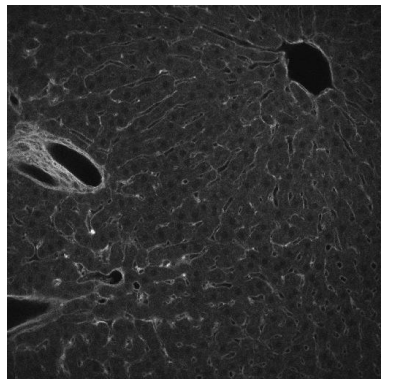

(a)

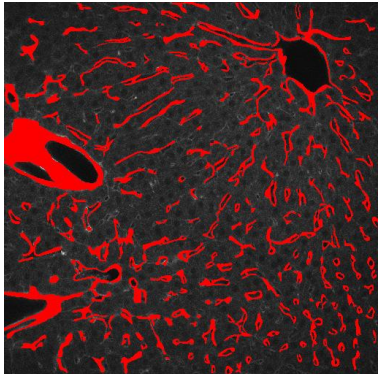

(b)

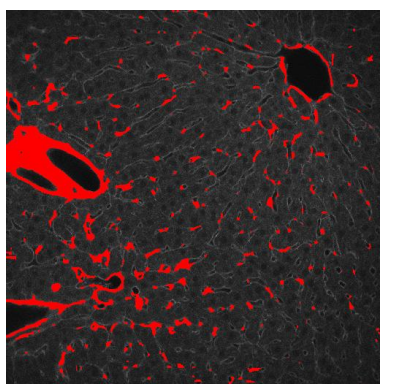

(e)

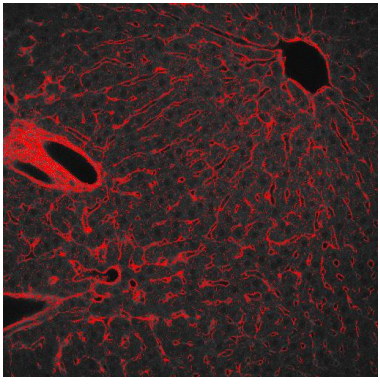

(c)

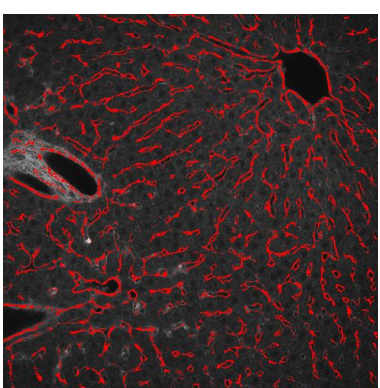

(f)

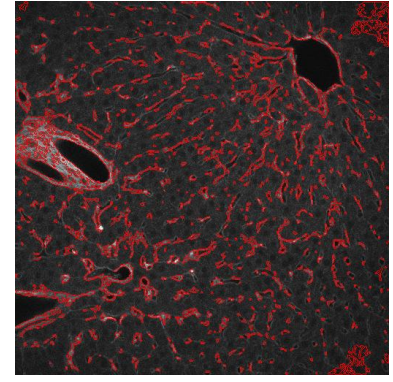

(d)

Figure 9. Comparison of the segmentation of $I_{z_{81}}$ in Data-IV by the proposed method and other methods. (a) the original image, (b) reference segmentation, (c) steerableJ segmentation outcome, (d) output image from region competition plugin, (e) Squassh segmented image, (f) outcome of the proposed method with tubule boundary only 